\title{
EQUIPOTENTIAL SURFACES FOR UNIFORMLY CHARGED SYMMETRIC POLYHEDRAL CONDUCTORS
}

This paper was downloaded from TechRxiv (https://www.techrxiv.org).

\section{LICENSE}

CC BY 4.0

SUBMISSION DATE / POSTED DATE

21-06-2021 / 23-06-2021

CITATION

CHAKRABORTY, Rishav (2021): EQUIPOTENTIAL SURFACES FOR UNIFORMLY CHARGED SYMMETRIC POLYHEDRAL CONDUCTORS. TechRxiv. Preprint.

https://doi.org/10.36227/techrxiv.14815386.v1

DOI

10.36227/techrxiv.14815386.v1 


\title{
EQUIPOTENTIAL SURFACES FOR UNIFORMLY CHARGED SYMMETRIC POLYHEDRAL CONDUCTORS
}

\author{
Rishav Chakraborty \\ Student \\ Kolkata, India \\ rishavchakraborty777@gmail.com
}

\begin{abstract}
In this paper the shape of equipotential surfaces of uniformly charged symmetric polyhedral conductors is discussed.It is observed that the shape of the equipotential surface is influenced more by the shape of the symmetric polyhedral conductor if it is evaluated at a smaller distance from that polyhedral conductor. As we move away from the conductor, the equipotential surface becomes similar to that due to a point source, which is spherical in shape. The distance between the symmetric polyhedral conductor and the first occurrence of its spherical equipotential surface has been mathematically derived.
\end{abstract}

\section{INTRODUCTION}

I was currently studying about equipotential surface and while studying a question arose in my mind about how the equipotential surfaces of a uniformly charged cubic conductor will look like. I initially didn't find any direct answer to this question on the Internet. So I worked on it myself.

Now, we know that for a uniformly charged conductor all charges reside on its surface.

(for simplicity one face of the cube is considered)

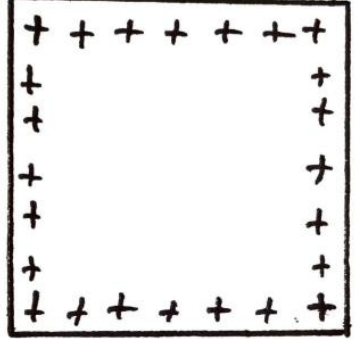

Fig.1. A face of the uniformly charged cubic conductor showing the distribution of charges when uniformly charged.
Equipotential surface is any surface that has an equal value of potential (in our case it will be electric potential) at every point on it. The surface of a uniformly charged conductor is an equipotential surface.

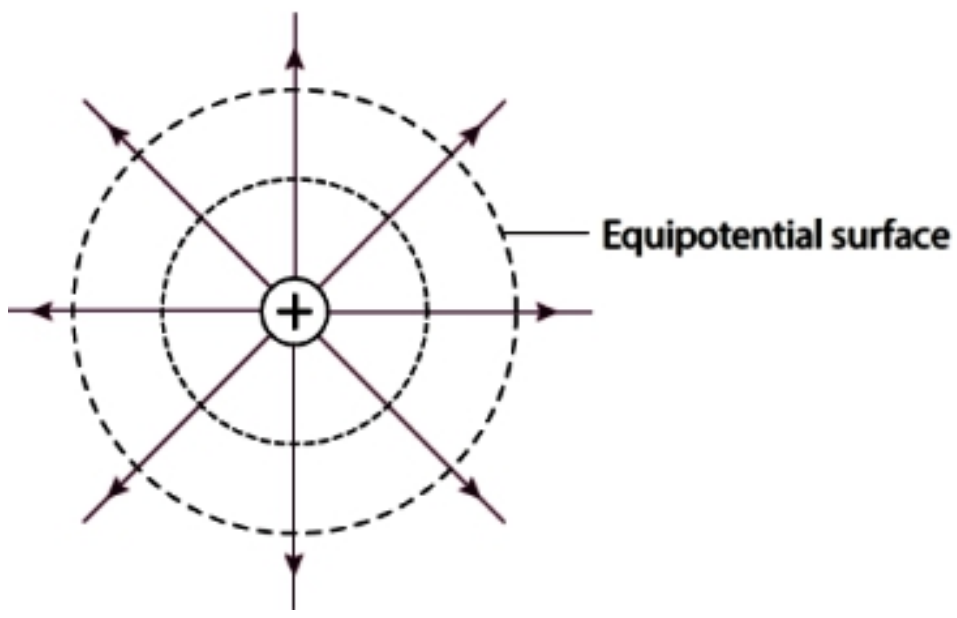

Fig.2. The equipotential surface due to a point charge.

\section{RESEARCH GAP}

In previous research papers[1] the concept of the curvature of equipotential surfaces at sharp bends for cubical conductor has been discussed. No theory regarding the equipotential surface of other polyhedral conductors has been proposed. The concept of the curvature of equipotential surface at sharp bends of uniformly charged conductors and it becoming spherical at far fields has been explained using concepts which might be unfamiliar to high school students. The distance between the conductor and the first occurrence of its spherical equipotential surface has never been derived. Anyone with basic knowledge of electrostatics will be able to understand the concepts proposed in this paper. 


\section{THE CURVATURE OF EQUIPOTENTIAL SURFACES AT SHARP BENDS OF UNIFORMLY CHARGED SYMMETRIC POLYHEDRAL CONDUCTORS}

Each face of the uniformly charged symmetric polyhedral conductor is like a charged conducting surface and for a charged conducting surface the electric fields lines due to it are normal to its surface. The charges at the sharp bends and corners have their electric field lines radially outwards.

If the face of the uniformly charged polyhedral conductor is divided into individual points, then each point will behave as point charge with its electric field lines projecting radially outwards. As the nature of the electric field (positive) is same there will be repulsion between the electric field lines of the individual points.

One side of the face of the conductor is considered for electric field lines as inside the charged conductor the net electric field is always zero.
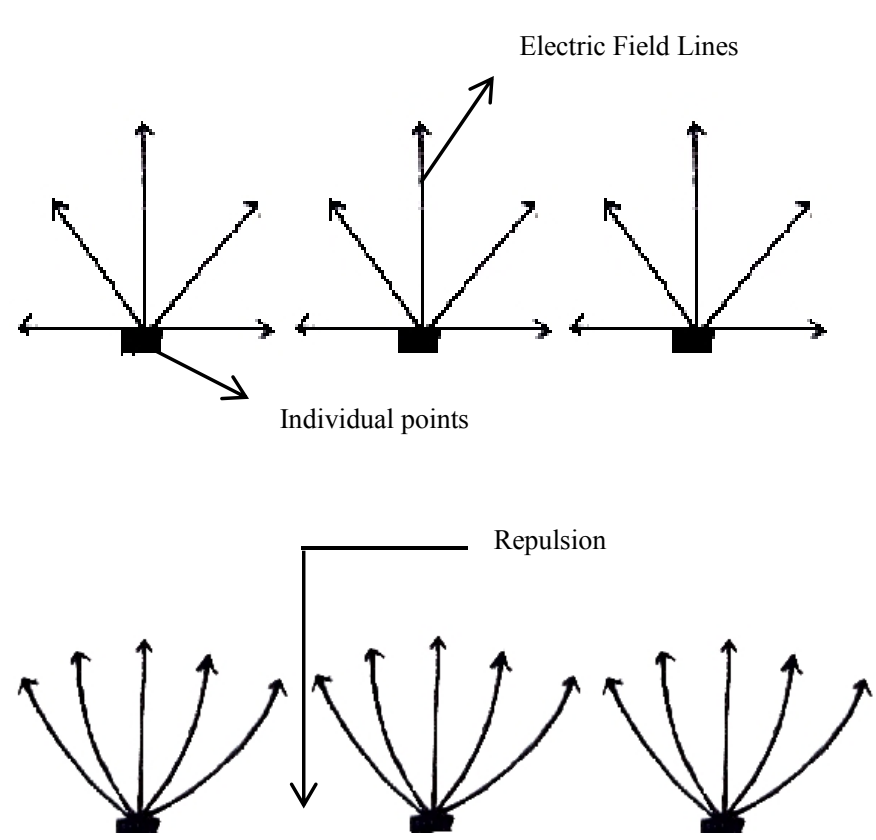

Repulsion between the electric field lines results in them bending away from each other as the individual points get closure.

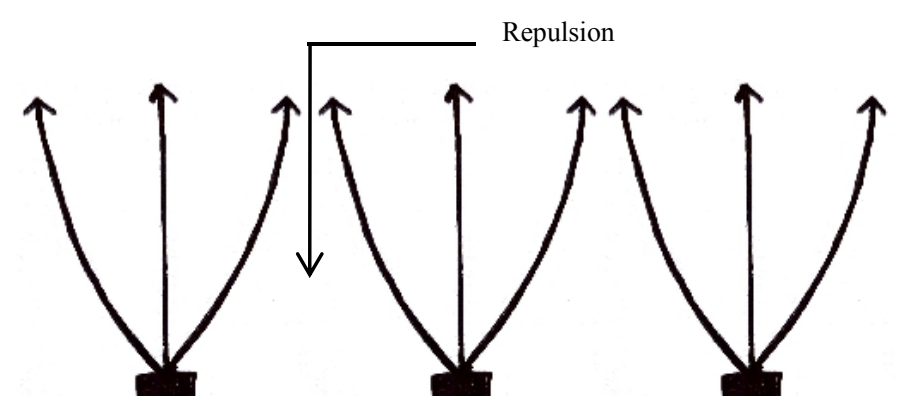

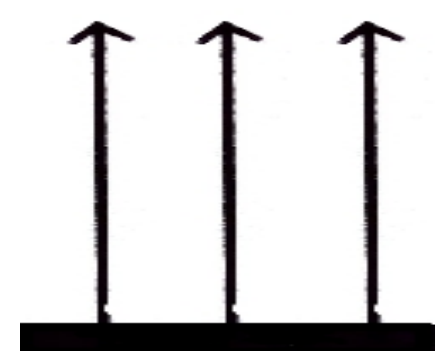

When the points get close enough the repulsion increases so much that from each individual point a single electric field line projects out perpendicular to the surface thus formed by bringing the individual points together.

Electric field lines at the sharp bends of the charged polyhedral conductor:
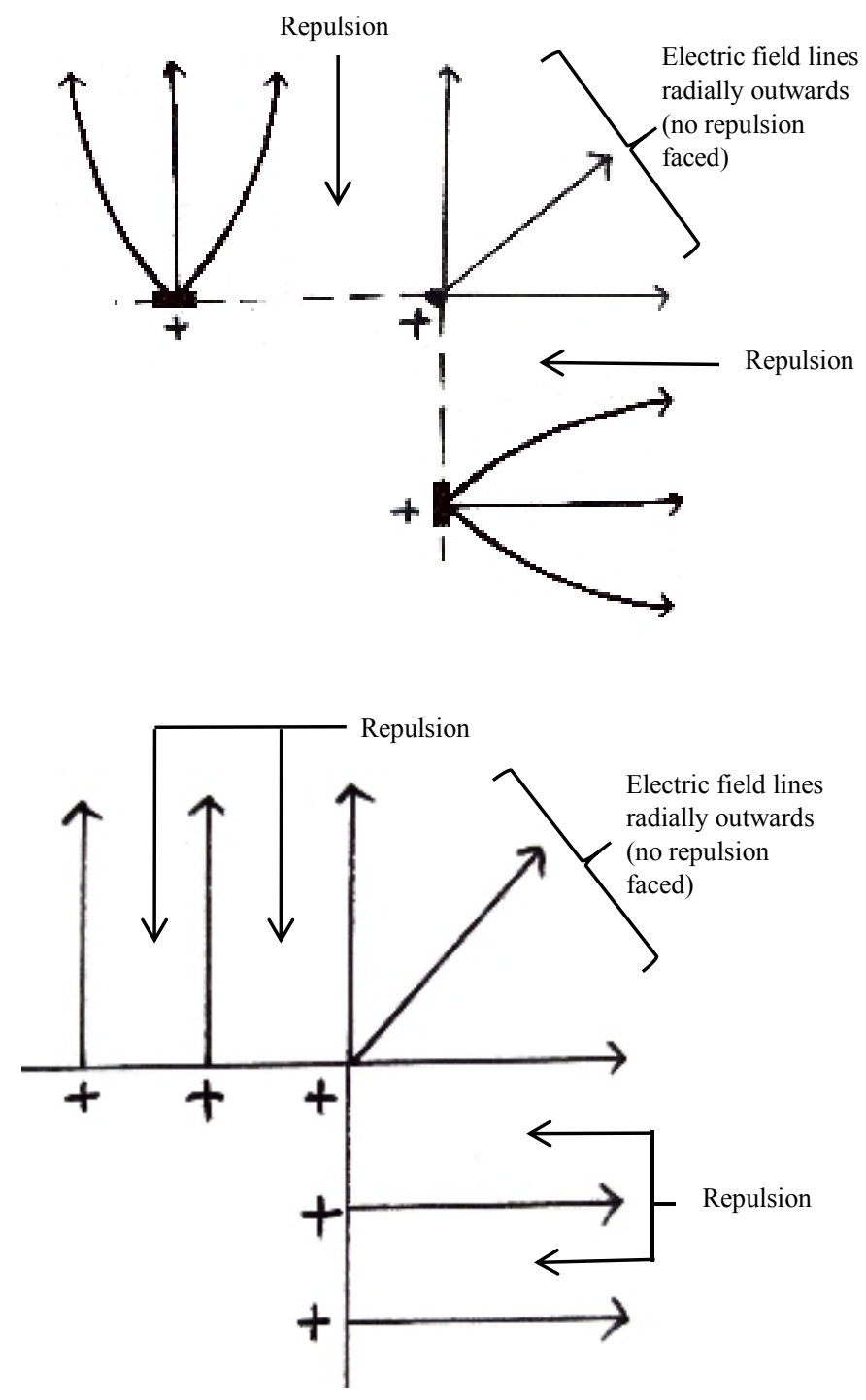
We know that:

$$
\mathrm{E}=-\frac{d V}{d x}
$$

where $\mathrm{V}$ is the electric potential, $\mathrm{E}$ is the electric field due to the charge and $\mathrm{x}$ is the distance from the charge.

For equipotential surfaces $V$ is constant, so $d V=0$

$$
\begin{gathered}
\mathrm{dV}=-\mathrm{Edx} \\
0=-\mathrm{Edx} \\
0=\mathrm{dx}
\end{gathered}
$$

If an electric field exists due to a uniformly charged conductor then the distance between its charged surface and its equipotential surface needs to be constant.

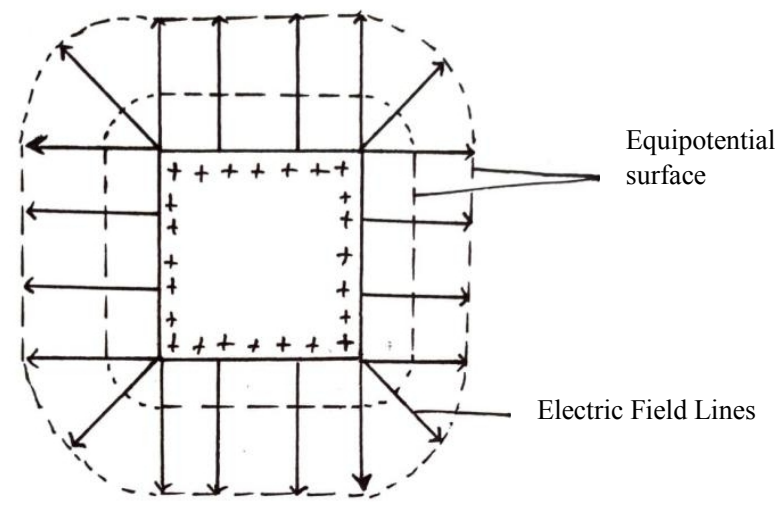

ELECTRIC FIELD AND EQUIPOTENTIAL SURFACE DUE TO A UNIFORMLY

CHARGED CUBIC CONDUCTOR

(for simplicity one face of the cube is considered)

The conductor's surface is itself an equipotential surface and as we move away from the conductor's surface, more cubical equipotential surfaces are formed with curved vertices. And with increase in distances the vertices curve to such an extent that the equipotential surface then resembles a spherical equipotential surface.
Equipotential surface

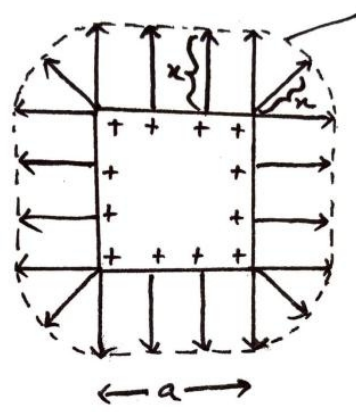

$a=$ edge of the cube, $x=$ distance between the cube's charged surface and the equipotential surface.

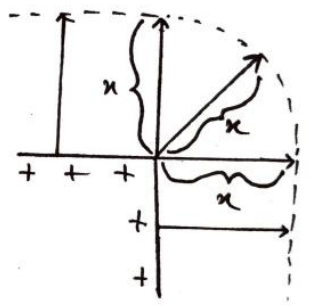

In the above figure, we can see that at the vertex part the equipotential surface looks like the arc of a quadrant of a circle of radius $\mathrm{x}$.

Now lets consider a symmetric tetrahedral conductor:

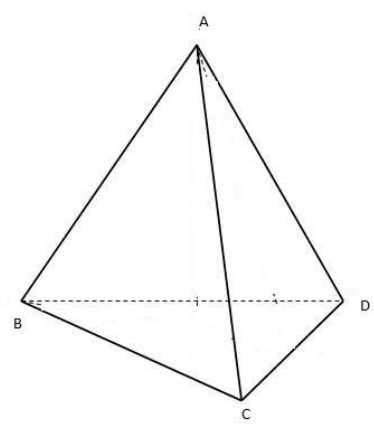




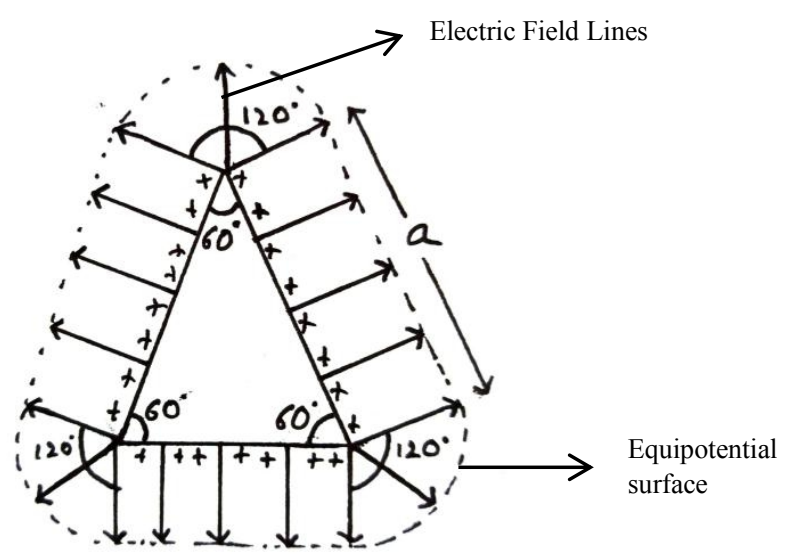

ELECTRIC FIELD AND EQUIPOTENTIAL SURFACE DUE TO A UNIFORMLY CHARGED SYMMETRIC TETRAHEDRAL CONDUCTOR

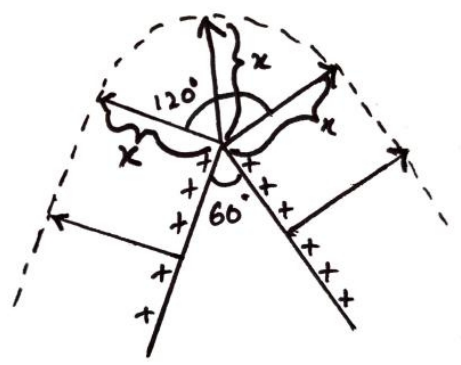

$\mathrm{a}=$ edge of the tetrahedron, $\mathrm{x}=$ distance between the tetrahedron's charged surface and the equipotential surface

In the above figure, we can see that at the vertex part the equipotential surface looks like the arc of a sector of a circle of radius $\mathrm{x}$ subtending an angle of 120 degrees.

In a research paper on "The Electric Field of a Uniformly Charged Non-Conducting Cubic Surface"[1] it was concluded that "The electric field magnitude can diverge to infinity for a charge distribution that has a sharp bend like the edges of the uniformly charged cubic surface. This can happen even though the charge density does not itself diverge in magnitude near an edge or vertex, as is the case for a charged conducting surface with a sharp bend.".

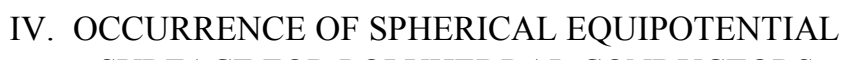
SURFACE FOR POLYHEDRAL CONDUCTORS

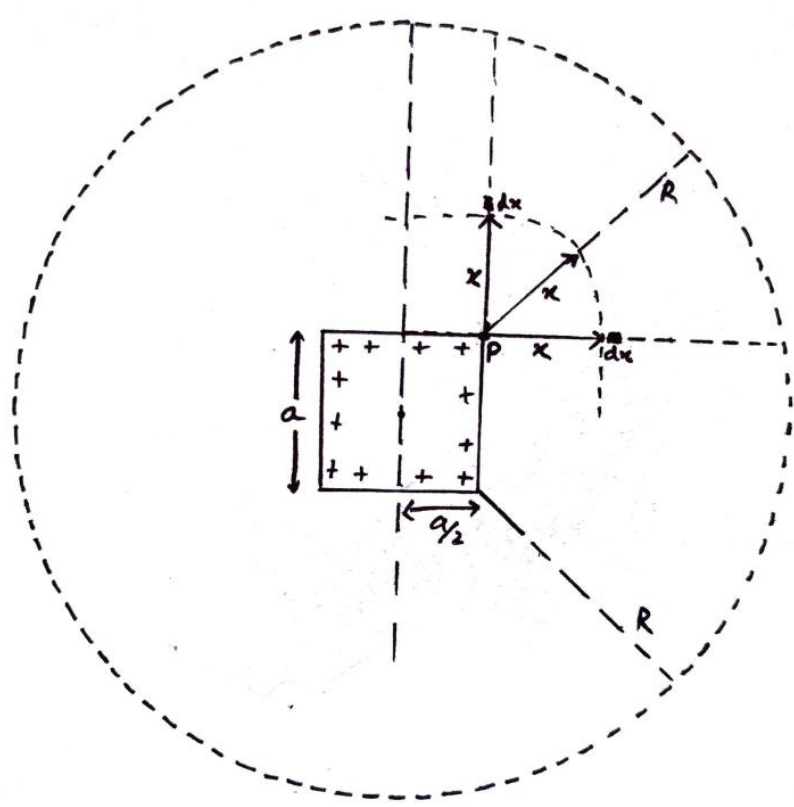

a.

$\mathrm{X}=$ radius of the small quadrant at the the vertices

$\mathrm{R}=$ radius of the large quadrant of the spherical equipotential surface

As $\mathrm{x}$ increases from from its initial point $\mathrm{P}$ on the vertex to $\mathrm{R}$, from the perspective of the increasing equipotential surfaces the cube shrinks in size and the point $\mathrm{P}$ shrinks from zero to $\mathrm{a} / 2$ for the spherical equipotential surface.

As $\mathrm{x}$ increases from zero (taken from a vertex ' $\mathrm{P}$ ') to $\mathrm{R}$ by a length of $d x$ (very small change in $\mathrm{x}$ ), the area of the small quadrant increases and finally becomes equal to the area of the large quadrant.

Area of the small quadrant(say 'Ax') $=\frac{\pi x^{2}}{4}$

$d A x=\frac{\pi x d x}{2} \longrightarrow \quad \begin{aligned} & \text { small change in area of } \\ & \text { quadrant }\end{aligned}$

Area of the large quadrant(say 'AR') $=\frac{\pi R^{2}}{4}$

Also, $\quad \mathrm{AR}=\int_{0}^{\mathrm{R}} d A x$ 
Rate of change of $\mathrm{x}$ from zero to $\mathrm{R}$ is equal to that from zero to $\mathrm{a} / 2$.

$$
\begin{aligned}
& \therefore \mathrm{AR}=\int_{0}^{\mathrm{R}} d A x \\
& \mathrm{AR}_{\mathrm{R}}=\int_{0}^{\frac{\mathrm{a}}{2}} \frac{\pi x d x}{2} \\
& \mathrm{AR}=\frac{\pi}{2} \int_{0}^{\frac{\mathrm{a}}{2}} x d x \\
& \mathrm{AR}=\frac{\pi}{2}\left[\frac{x^{2}}{2}\right]_{0}^{\frac{\mathrm{a}}{2}} \\
& \mathrm{AR}=\frac{\pi}{2} \cdot \frac{\mathrm{a}}{8} \\
& \frac{\pi R^{2}}{4}=\frac{\pi}{2} \cdot \frac{\mathrm{a}}{8} \\
& R^{2}=\frac{\mathrm{a}^{2}}{4} \\
& R=\frac{a}{2}
\end{aligned}
$$

\section{b.}

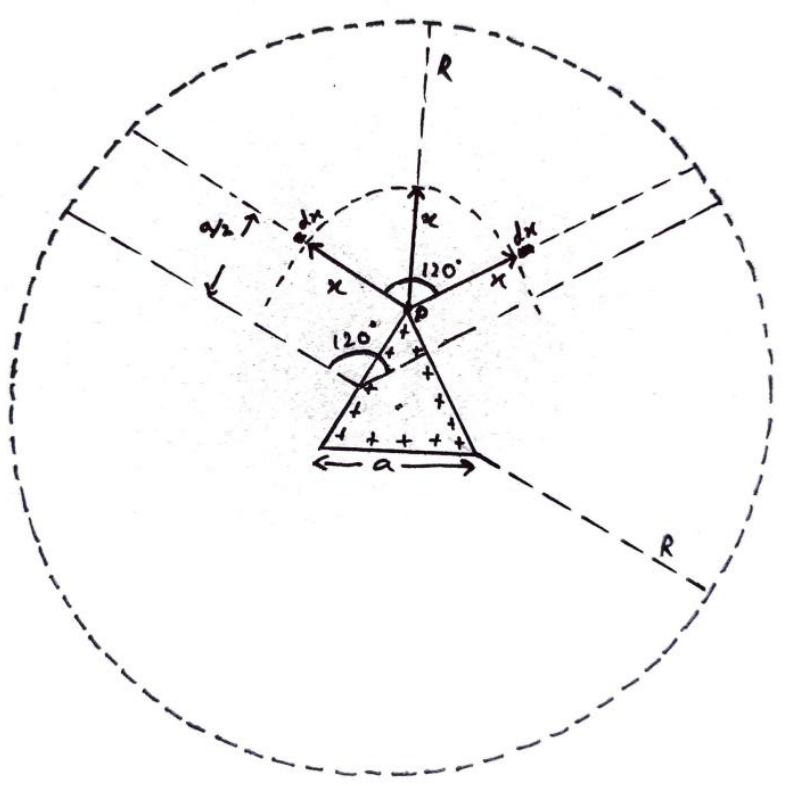

$\mathrm{X}=$ radius of the small sector at the the vertices

$\mathrm{R}=$ radius of the large sector of the spherical equipotential surface

As $\mathrm{x}$ increases from from its initial point $\mathrm{P}$ on the vertex to $\mathrm{R}$, from the perspective of the increasing equipotential surfaces the tetrahedron shrinks in size and the point $\mathrm{P}$ shrinks from zero to $\mathrm{a} / 2$ for the spherical equipotential surface.

As $\mathrm{x}$ increases from zero (taken from a vertex ' $\mathrm{P}$ ') to $\mathrm{R}$ by a length of $d x$ (very small change in $\mathrm{x}$ ), the area of the small sector increases and finally becomes equal to the area of the large sector.

$$
\begin{aligned}
& \text { Area of the small sector(say 'Ax') }=\frac{\pi x^{2}}{3} \\
& d A x=\frac{2 \pi x d x}{3} \longrightarrow \begin{array}{l}
\text { small change in area of } \\
\text { sector }
\end{array}
\end{aligned}
$$$$
\text { Area of the large sector(say 'AR') }=\frac{\pi R^{2}}{3}
$$

$$
\text { Also, } \quad \mathrm{AR}=\int_{0}^{\mathrm{R}} d A x
$$

Rate of change of $\mathrm{x}$ from zero to $\mathrm{R}$ is equal to that from zero to $\mathrm{a} / 2$. 


$$
\begin{aligned}
& \therefore \mathrm{AR}=\int_{0}^{\mathrm{R}} d A x \\
& \mathrm{AR}=\int_{0}^{\frac{\mathrm{a}}{2}} \frac{2 \pi x d x}{3} \\
& \mathrm{AR}=\frac{2 \pi}{3} \int_{0}^{\frac{\mathrm{a}}{2}} x d x \\
& \mathrm{AR}=\frac{2 \pi}{3}\left[\frac{x^{2}}{2}\right]_{0}^{\frac{\mathrm{a}}{2}} \\
& \mathrm{AR}=\frac{2 \pi}{3} \cdot \frac{\mathrm{a}}{8} \\
& \frac{\pi R^{2}}{3}=\frac{2 \pi}{3} \cdot \frac{\mathrm{a}}{8} \\
& R=\frac{\mathrm{a}}{2} \\
& R^{2}=\frac{\mathrm{a}^{2}}{4} \\
& R
\end{aligned}
$$

\section{THEORY}

For a uniformly charged symmetric polyhedral conductor where length of a side is ' $a$ ', its equipotential surface curves at the vertices as we move away from it and finally becomes similar to a spherical equipotential surface and the diagonal distance between its vertices and the first occurrence of its spherical equipotential surface is equal to half of ' $a$ '.

Let ' $R$ ' be the diagonal distance between the vertices of the uniformly charged symmetric polyhedral conductor and its first occurrence of its spherical equipotential surface.

Then,

$$
\mathrm{R}=\frac{\mathrm{a}}{2}
$$

\section{RELATION BETWEEN R AND THE NUMBER OF VERTICES AND FACES OF A POLYHEDRAL CONDUCTOR}

The surface area of a polyhedron is equal to the sum of the area of all of its faces.

Let the number of faces of the uniformly charged symmetric polyhedral conductor be ' $\mathrm{f}$ '

Surface area $=\mathrm{f} *$ area

and the area of one face of a symmetric polyhedron

$$
=\frac{1}{2} * \text { perimeter } * \text { apothem }
$$

(apothem $=$ distance between the centre of a face of a polyhedron and one of its sides)

Let the number of sides of one face of a symmetric polyhedron be ' $s$ ' and the no of vertices of one face of a symmetric polyhedron be ' $\mathrm{v}$ '.

For a face of a symmetric polyhedron, $\mathrm{v}=\mathrm{s}$

Perimeter of one face of a symmetric polyhedron $=\mathrm{s} * \mathrm{a}$

$$
\text { area }=\frac{1}{2} * \mathrm{v} * \mathrm{a} * \text { apothem }
$$

$$
=\mathrm{v} * \mathrm{a}
$$

Therefore, surface area $=\mathrm{f} * \frac{1}{2} * \mathrm{v} * \mathrm{a} *$ apothem

Keeping surface area constant (say 'c')

$$
\begin{aligned}
& \mathrm{c}=\mathrm{f} * \frac{1}{2} * \mathrm{v} * \mathrm{a} * \text { apothem } \\
& \mathrm{a}=\frac{\mathrm{c}}{\mathrm{f} * \frac{1}{2} * \mathrm{v} * \text { apothem }}
\end{aligned}
$$

Therefore, $a \propto \frac{1}{\mathrm{f}} \& \mathrm{a} \propto \frac{1}{\mathrm{v}}$

And, $\mathrm{R} \propto \mathrm{a}$

Therefore, $R \propto \frac{1}{\mathrm{f} * \mathrm{v}}$

\section{Comparing $\mathbf{R}$ for a cubic and a tetrahedral conductor:}

Keeping the surface area of both the polyhedral conductors equal to 1 ,

$\mathrm{v}$ and $\mathrm{f}$ for cubic conductor is 8 and 6 respectively $\mathrm{v}$ and $\mathrm{f}$ for tetrahedral conductor is 4 and 4 respectively $(\mathrm{v} * \mathrm{f})$ for cubic conductor is 48 
$(\mathrm{v} * \mathrm{f})$ for tetrahedral conductor is 16

$\mathrm{v} * \mathrm{f}($ cubic $)>\mathrm{v} * \mathrm{f}($ tetrahedral $)$

As

$$
R \propto \frac{1}{f * v}
$$

Therefore, R (cubic) < R (tetrahedral)

Now, lets find that mathematically

Keeping the surface area of the both polyhedral conductors equal to 1 ,

a for cubic conductor is 0.408

a for tetrahedral conductor is approx. 0.76

$\mathrm{R}($ cubic $)=\mathrm{a} / 2=(0.408) / 2=0.204$

$\mathrm{R}($ tetrahedral $)=\mathrm{a} / 2=(0.76) / 2=0.38$

$\mathrm{R}$ (cubic) $<\mathrm{R}$ (tetrahedral) [proved]

\section{THEORETICAL PROOF}

$\mathrm{R}$ is inversely proportional to $\mathrm{V}$.

If $\mathrm{v}=\infty$,

Then, $\mathrm{R}=0$, according to the theory.

A sphere is a polyhedron where $\mathrm{v}=\infty$ and its vertices form its spherical surface.

And for a uniformly charged spherical conductor its first spherical equipotential surface is its own surface, hence the $\mathrm{R}$ in this case becomes zero.

\section{CONCLUSION}

In this paper, I have explained how the equipotential surface is influenced by the shape of the symmetric polyhedral conductor and how it finally becomes spherical as it would be if the polyhedral conductor is replaced by a point charge placed at the centre of that conductor. As we move away from the polyhedral conductor the consecutive equipotential surfaces curve more at the sharp bends. A pattern is observed when the diagonal distance from the vertex of the conductor to the first occurrence of its spherical equipotential surface for all symmetric polyhedral conductors is mathematically calculated which is half of the length of its side.

\section{REFERENCES}

[1] Kaitlin McCreery and Henry Greenside, "The Electric Field of a Uniformly Charged Non-Conducting Cubic Surface", Department of Physics, Duke University, Durham NC 27708-0305, 2016, pp. 33.

[2] S.L.Arora, "New Simlified Physics", $12^{\text {th }}$ ed, vol. 1, Dhanpat Rai \& Co., 2021, pp. $2.14-2.16$. 